\title{
Critical point anomalies include expansion shock waves
}

\author{
N. R. Nannan, ${ }^{1, \text { a) }}$ A. Guardone, ${ }^{2, b)}$ and P. Colonna ${ }^{3, c)}$ \\ ${ }^{1}$ Mechanical Engineering Discipline, Anton de Kom University of Suriname, Leysweg 86, \\ PO Box 9212, Paramaribo, Suriname and Process and Energy Department, \\ Delft University of Technology, Leeghwaterstraat 44, 2628 CA Delft, The Netherlands \\ ${ }^{2}$ Department of Aerospace Science and Technology, Politecnico di Milano, Via La Masa 34, \\ 20156 Milano, Italy \\ ${ }^{3}$ Propulsion and Power, Delft University of Technology, Kluyverweg 1, 2629 HS Delft, \\ The Netherlands
}

(Received 9 September 2013; accepted 9 January 2014; published online 13 February 2014)

From first-principle fluid dynamics, complemented by a rigorous state equation accounting for critical anomalies, we discovered that expansion shock waves may occur in the vicinity of the liquid-vapor critical point in the two-phase region. Due to universality of near-critical thermodynamics, the result is valid for any common pure fluid in which molecular interactions are only short-range, namely, for so-called 3-dimensional Ising-like systems, and under the assumption of thermodynamic equilibrium. In addition to rarefaction shock waves, diverse non-classical effects are admissible, including composite compressive shock-fan-shock waves, due to the change of sign of the fundamental derivative of gasdynamics. (C) 2014 AIP Publishing LLC. [http://dx.doi.org/10.1063/1.4863555]

Shock waves are observed in all typical states of matter, namely, in single- or multi-phase conditions. These finite-amplitude waves propagate at speed higher than the local speed of sound, i.e., at supersonic speed, and produce a sudden variation of the thermo-physical state of matter; in gases, the thickness of the transition front is comparable to the molecular mean free path (close to $20 \mathrm{~nm}$ in air in standard conditions). In most situations, shock waves in gaseous, liquid, and solid media are of the compressive type only, namely, produce an increase of pressure, temperature, and density of the media, as observed in explosions or around aircraft flying at supersonic speed in the atmosphere. Non-classical shock waves of the expansive type have been experimentally observed during allotropic phase changes in steel. ${ }^{1}$ Thompson et al. ${ }^{2}$ and the sources mentioned in Ref. 2 document, among many liquid-vapor phase transition phenomena, expansive liquid-evaporation waves. Non-classical rarefaction shocks are believed to be admissible also in a current-carrying plasma. ${ }^{5}$

Ultimately, the nature of shock waves, i.e., whether the shock wave is compressive or expansive, is determined by the value of the fundamental derivative of gas dynamics $\Gamma$,

$$
\Gamma \equiv 1+\frac{\rho}{c}\left(\frac{\partial c}{\partial \rho}\right)_{s},
$$

with $\rho, c$, and $s$ density, sound speed, and entropy per unit mass, respectively. ${ }^{3,4}$ The fundamental derivative of gas dynamics is always positive for common fluids like nitrogen and water in the idealgas phase, assuming a constant heat capacity. As a consequence, in common fluids and in dilute conditions, only compression shock waves can be observed. Particularly, the negative sign of $\Gamma$ allows for the admissibility of expansive shock waves and even composite waves, such as compressive shock-fans or expansive fan-shock-fans, see, for example, Ref. 4. These phenomena are referred to as non-classical gas-dynamic effects and possibly include stationary rarefaction shock waves.

\footnotetext{
a)Electronic mail: ryan.nannan@uvs.edu

b) Electronic mail: alberto.guardone@ polimi.it

c) Electronic mail: p.colonna@tudelft.nl
} 
The present study stemmed from three observations. First, experiments show that some thermodynamic and transport properties, including the specific heats, diverge to large values as the critical point of pure fluids is approached. ${ }^{6-8}$ This also implies divergence of the fundamental derivative of gas dynamics $\Gamma$. Experimental observations report on the divergence of the nonlinear acoustic parameter, which is proportional to $\Gamma$, in the vicinity of the critical point ${ }^{9}$ Second, the three available theoretical studies considering the gasdynamics in the close-to-critical region provide values of $\Gamma$ only for single-phase fluid states. ${ }^{10-12}$ These studies confirmed that $\Gamma$ diverges at the critical point. In particular, Ref. 12 shows that $\Gamma$ diverges to positive infinity as the critical point is approached from the single-phase side. Third, no experimental observation of close-to-critical flows is available, with the only exception of the experiments of Borisov et al. ${ }^{10}$ which unfortunately are at odds with Emanuel's prediction ${ }^{12}$ due to a computational error in Ref. 10.

This study considers the value of $\Gamma$ and gas-dynamic effects related to its anomalous behavior in the single- and two-phase vapor-liquid critical region. To this purpose, state-of-the-art critical point models are adopted, under the hypothesis of thermodynamic equilibrium and assuming that the two phases are homogeneously and finely dispersed. The influence of surface tension is neglected since its value is very low within the critical region, where the fluid can be represented as an interdispersed mist with no physically meaningful distinction between the liquid and vapor phase.

In the following, conditions leading to the occurrence of non-classical waves in the critical point region are briefly discussed and the pre- and post-wave states of an exemplary rarefaction shock wave and of a mixed shock-fan-shock wave in methane in close-to-critical conditions are reported and discussed.

The divergence of thermodynamic properties near the critical point is usually described in terms of scaling laws, since the Helmholtz free energy becomes non-analytic at the critical point and therefore classical equations of state, e.g., cubic equations of state, are unsuitable to model near-critical behavior. ${ }^{14,24}$ To determine properties in the near-critical state of pure fluids, including $\Gamma$, we used the scaled fundamental equation of state formulated by Balfour et al. ${ }^{15}$ see also Ref. 16 for the technical details. Such thermodynamic model is only applicable within the critical region of so-called 3-dimensional Ising-like systems, i.e., systems governed by short-range forces. Plasmas and vapor of metals and salts are therefore excluded.

By applying the scaled fundamental equation of state, we found that $\Gamma$ diverges to large positive values as the critical point is approached from the single-phase side. ${ }^{16}$ More specifically, along the dew-line as the critical point is approached,

$$
\lim _{T \uparrow T_{\mathrm{c}}} \Gamma\left(P^{\mathrm{sat}}(T), T\right) \propto \lim _{T \uparrow T_{\mathrm{c}}}\left[\frac{T-T_{\mathrm{c}}}{T}\right]^{-\kappa}=\lim _{T \uparrow T_{\mathrm{c}}}-\frac{T-T_{\mathrm{c}}}{T}\left|\frac{T-T_{\mathrm{c}}}{T}\right|^{-(\kappa+1)} \rightarrow+\infty,
$$

where $P^{\text {sat }}(T)$ is the saturation pressure at temperature $T$ and the subscript c indicates critical point values. The critical exponent $\kappa$ of the fundamental derivative of gas dynamics is computed as a combination of the isochoric critical exponent $\alpha$, the saturation critical exponent $\beta$, and the critical isotherm exponent $\delta$, as $\kappa=\beta(\delta+1)=1-\alpha=0.890 \pm 0.003$. Therefore, $\kappa$ is a universal constant, since $\alpha, \beta$, and $\delta$ are universal constants for all fluids belonging to the class of 3-dimensional Ising-like systems. Similarly, along the critical isochore in the single-phase region,

$$
\lim _{T \downarrow T_{\mathrm{c}}} \Gamma\left(\rho_{\mathrm{c}}, T\right) \propto \lim _{T \downarrow T_{\mathrm{c}}} \frac{T-T_{\mathrm{c}}}{T}\left|\frac{T-T_{\mathrm{c}}}{T}\right|^{-(\kappa+1)} \rightarrow+\infty .
$$

The divergence of $\Gamma$ in the single-phase critical region is in agreement with Emanuel's result. ${ }^{12}$ In the vapor-liquid equilibrium region, our calculations yield that along the critical isochore, as the critical point is approached, $\Gamma$ diverges to negative infinity as

$$
\lim _{T \uparrow T_{\mathrm{c}}} \Gamma\left(\rho_{\mathrm{c}}, T\right) \propto \lim _{T \uparrow T_{\mathrm{c}}} \frac{T-T_{\mathrm{c}}}{T}\left|\frac{T-T_{\mathrm{c}}}{T}\right|^{-(\kappa+1)} \rightarrow-\infty .
$$

The above power-law dependence is also universal, i.e., valid for any pure fluid. 

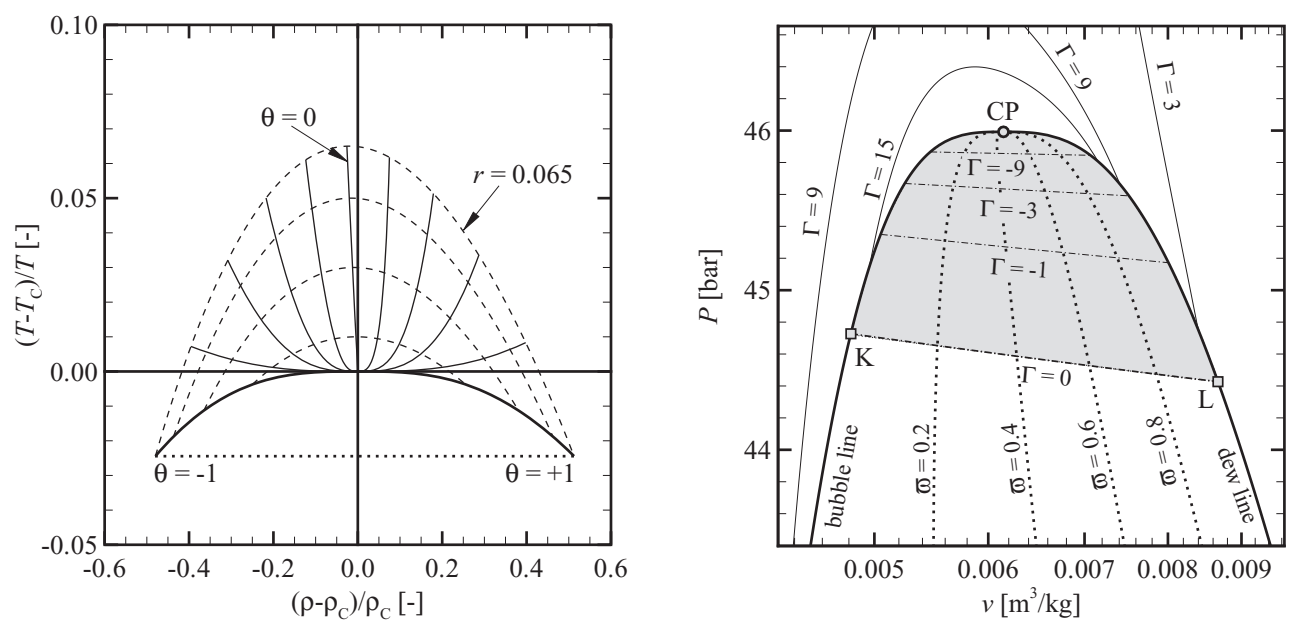

FIG. 1. Left: Definition of the critical region in terms of the parametric coordinates $\theta$ and $r$, valid for the universality class of 3-dimensional Ising-like systems. Right: Lines of constant fundamental derivative of gas dynamics $\Gamma$ in the vapor-liquid equilibrium region of methane near its critical point, illustrated in the pressure-specific volume diagram. The equation of state is that of Kurumov et al. ${ }^{13}$ The shaded region is the $\Gamma<0$ region. The gray circle indicates the vapor-liquid critical point. The dotted lines are lines of constant vapor mass-fraction $\varpi$.

The consequence of negative $\Gamma$ values in the two-phase vapor-liquid equilibrium region is that expansive shock waves are admissible, among several other anomalous phenomena including mixed or split waves, in the two-phase critical point region.

In Figure 1 (left), the critical point region according to the model proposed by Kurumov et al. ${ }^{13}$ is described in terms of so-called parametric variables $r$ and $\theta$, where $r$ gives the "distance" of a given thermodynamic state with respect to the critical point and the variable denoted by $\theta$ gives the location of the thermodynamic state on a line of constant- $r$, such that $\theta=-1$ is the dew-line and $\theta$ $=+1$ is the bubble-line $(-1 \leq \theta \leq+1)$. In Figure 1 (right), the value of $\Gamma$ is depicted for methane $\left(\mathrm{CH}_{4}\right)$.

Since the exponent $\kappa$ introduced here in (2)-(4) is a universal critical exponent, we conclude that expansion shock waves are physically admissible in the vicinity of the critical point region for all typical fluids, including, e.g., argon and other noble gases, simple fluids such as water, nitrogen, or carbon dioxide, and organic compounds such as alkanes and ethers, olefins, and paraffins.

In order to determine the fluid states complying with an admissible rarefaction shock wave, the conservation laws of mass, momentum, and total energy are applied across the shock front. Under the assumption of thermodynamic equilibrium, the relevant equations are the Rankine-Hugoniot jump conditions, that is a nonlinear system of three algebraic equations relating the pre-shock values of the density, pressure, and fluid velocity (state 1) to the corresponding post-shock values (state 2 ), for a given shock speed. Admissibility conditions are subsequently verified in order to ensure mechanical stability of the shock front, and to break the time symmetry. Admissibility conditions are: (i) the speed-ordering relation, i.e., $\mathrm{Ma}_{1} \geq 1 \geq \mathrm{Ma}_{2}$ (the Mach number, Ma, is the ratio of the flow speed in a frame of reference moving with the shock wave with respect to the local value of the sound speed), and (ii) the second law of thermodynamics which imposes an entropy increase across the shock wave, i.e., $s_{2} \geq s_{1}$, see Refs. 17 and 18; note that $s_{2} \sim s_{1}$ for weak shock waves.

The solution of the Rankine-Hugoniot system of equations is usually visualized in the specific volume-pressure thermodynamic plane, where all possible post-shock values of the pressure can be drawn as a function of the post-shock specific volume, for given values of the pre-shock pressure and specific volume. This locus is the Hugoniot curve or shock adiabat. For a given shock speed, the unique post-shock state is located at the intersection of the Hugoniot curve and the so-called Rayleigh line. The latter is obtained from mass and momentum conservation across the shock surface. The speed-ordering relation and the entropy inequality are then employed to assess if such a shock wave is admissible. More specifically, if the Rayleigh line connecting the pre- and post-shock states in a 
TABLE I. Thermodynamics conditions leading to the formation of the single rarefaction shock wave in Figure 3 and of the composite wave in Figure 4. The Mach number is computed in the wave reference.

\begin{tabular}{|c|c|c|c|c|c|c|}
\hline & State & $\begin{array}{c}P \\
\text { (bar) }\end{array}$ & $\begin{array}{c}v \\
\left(\mathrm{~m}^{3} / \mathrm{kg}\right)\end{array}$ & $\begin{array}{c}T \\
(\mathrm{~K})\end{array}$ & $\Gamma$ & Ma \\
\hline \multirow[t]{2}{*}{ Rarefaction shock wave } & 1 & 45.50 & 0.00764 & 190.21 & -2.30 & 1.0017 \\
\hline & 2 & 43.74 & 0.00809 & 188.95 & 0.379 & 1.0000 \\
\hline \multirow[t]{4}{*}{ Mixed compression wave } & 1 & 44.00 & 0.00750 & 189.13 & 0.288 & 1.002 \\
\hline & 2 & 44.73 & 0.00732 & 189.66 & -0.198 & 1.000 \\
\hline & 3 & 45.34 & 0.00717 & 190.09 & -1.400 & 1.000 \\
\hline & 4 & 45.96 & 0.00702 & 190.53 & 12.00 & 0.692 \\
\hline
\end{tabular}

specific volume-pressure diagram is located completely above the corresponding Hugoniot curve, the wave is a compression shock wave. Conversely, if the Rayleigh line connecting the pre- and post-shock state is located completely below the Hugoniot curve in between these aforesaid states, the wave is an expansion shock wave, see Ref. 19. These two conclusions additionally require that the derivative $(\partial P / \partial e)_{v}$, with $e$ internal energy per unit mass, is positive at the post-shock state. ${ }^{20}$ According to experimental evidence, this condition is fulfilled in the thermodynamic domain of interest here. The post-shock state is determined without considering the effects of viscosity and thermal conductivity, since it is independent of the dissipative mechanism, even if the shock wave is dispersed, see Ref. 21.

Table I reports the fluid states computed with the above procedure and resulting in a rarefaction shock wave and in a composite compression fan-shock-fan wave. Fluid states are depicted in Figure 2 in the volume-pressure thermodynamic plane.

A near-critical rarefaction shock wave can be appreciated with the aid of Figure 3, depicting, in addition to iso- $\Gamma$ lines of negative value, also the pre- and post-shock states in the pressure-specific volume state diagram of an exemplary fluid, namely, methane. Figure 3 presents an illustrative sketch of the same two-phase shock wave of Figure 2 (left), in which the curvature of the isentropes, Hugoniot locus and dew line is exaggerated. In terms of dimensional values, the calculation performed with the scaled equation of state of Kurumov et al. ${ }^{13}$ for methane as a test fluid gives for pre-shock state 1 a pressure of 45.50 bars, a temperature of $190.21 \mathrm{~K}$, and a vapor fraction $\varpi \uparrow 1$. A possible post-shock state 2 corresponds to a pressure of 43.74 bars and a temperature of $188.95 \mathrm{~K}$. Moreover, state 2 is characterized by a post-shock Mach number equal to one and a vapor fraction of 0.763 .
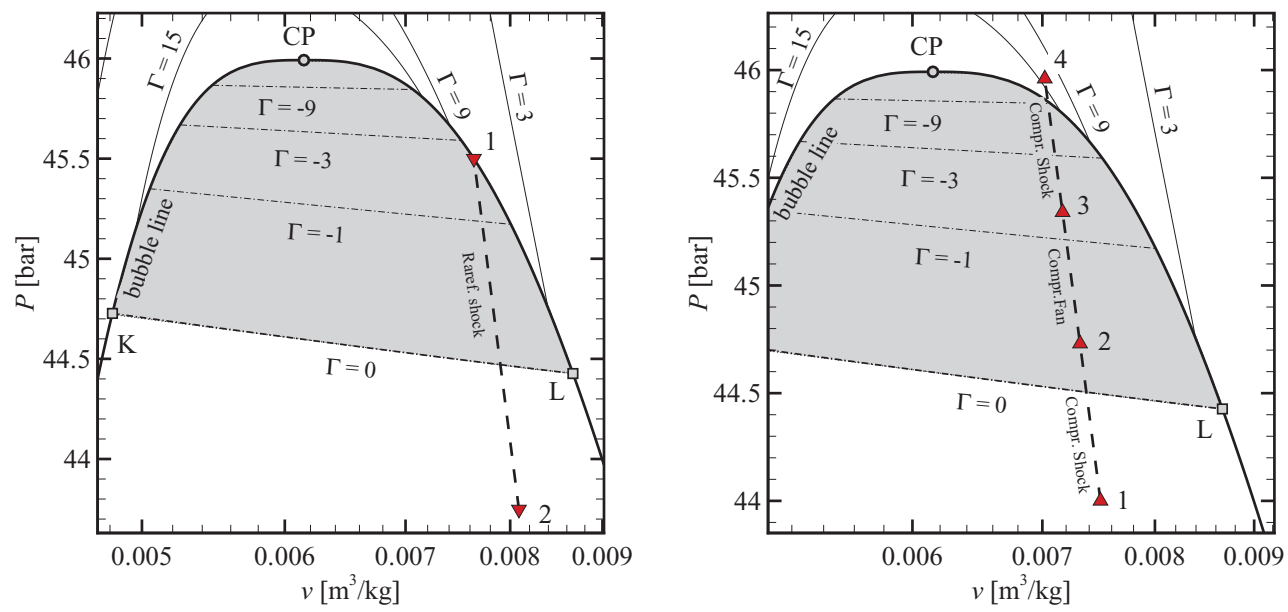

FIG. 2. Fluid state in the volume-pressure plane, see Table I, for the rarefaction shock wave described in Figure 3 (left) and the composite wave in Figure 4 (right). Note that the composite wave is a compressive wave. 

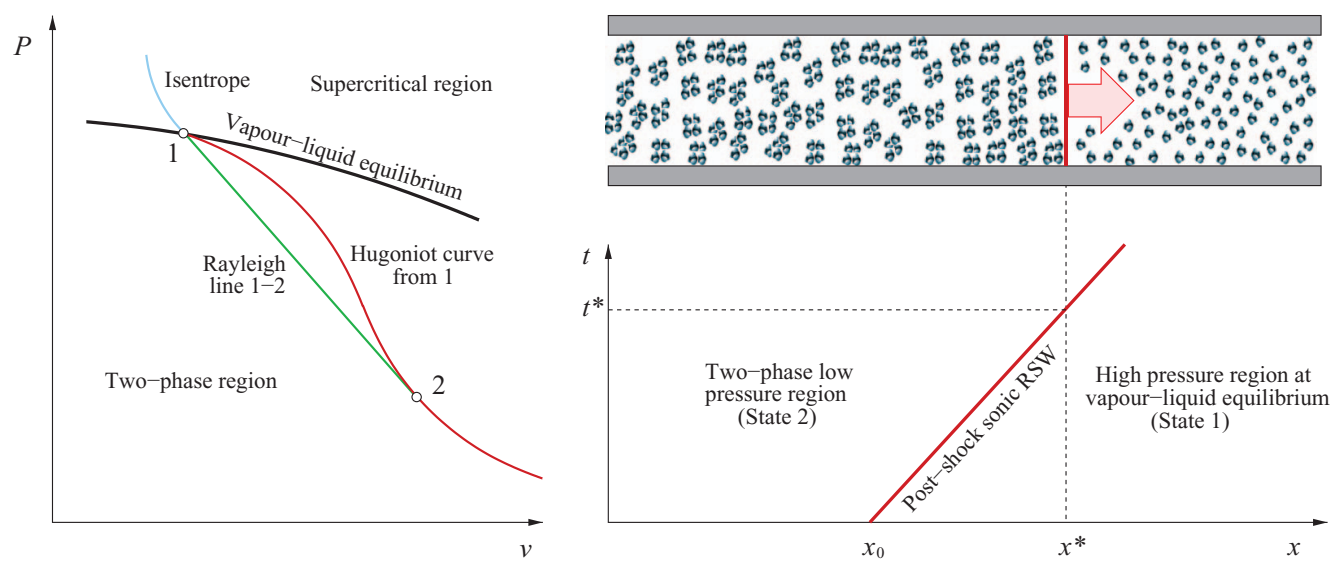

FIG. 3. Illustrative sketch, not to scale, showing the shock adiabat from state 1, its curvature, and the Rayleigh line connecting pre- and post-shock states 1 and 2 related to an admissible rarefaction shock wave according to Figure 2 (left). Also shown is the $x$ - $t$-plot and a snapshot of the flow field in a shock tube at time $t=t^{*}$ at the corresponding location. RSW is the abbreviation of rarefaction shock wave.

This shock wave originating at state 1 displays the largest possible pressure change, namely, 1.76 bars, and the associated pre-shock Mach number is 1.022, according to the procedure described in Refs. 19 and 22. The entropy jump equals $9.15 \times 10^{-5} \mathrm{~J} / \mathrm{kg} \mathrm{K}$. The fluid states are gathered in Table I.

A second example of an exotic wave field of the compressive type is illustrated in Figure 4. Here a composite compression wave is shown consisting of a non-classical compression shock wave with a post-shock Mach number of one, followed by a non-classical compression fan. At a certain thermodynamic state 3 , the wave speed is such that coalescence of the wave occurs and a second shock wave is admitted causing phase transition $3 \rightarrow 4$. For methane an example of such a composite wave field features the calculated states reported in Table I. Note that state 4 is in the single-phase dense gas region and that a very small entropy difference is observed across the two (weak) sonic shocks, namely, $s_{2}-s_{1}=2.30 \times 10^{-5} \mathrm{~J} / \mathrm{kg} \mathrm{K}$ and $s_{4}-s_{3}=1.55 \times 10^{-5} \mathrm{~J} / \mathrm{kg} \mathrm{K}$.

Similar qualitative results can be obtained with other fluids, such as carbon dioxide and water.
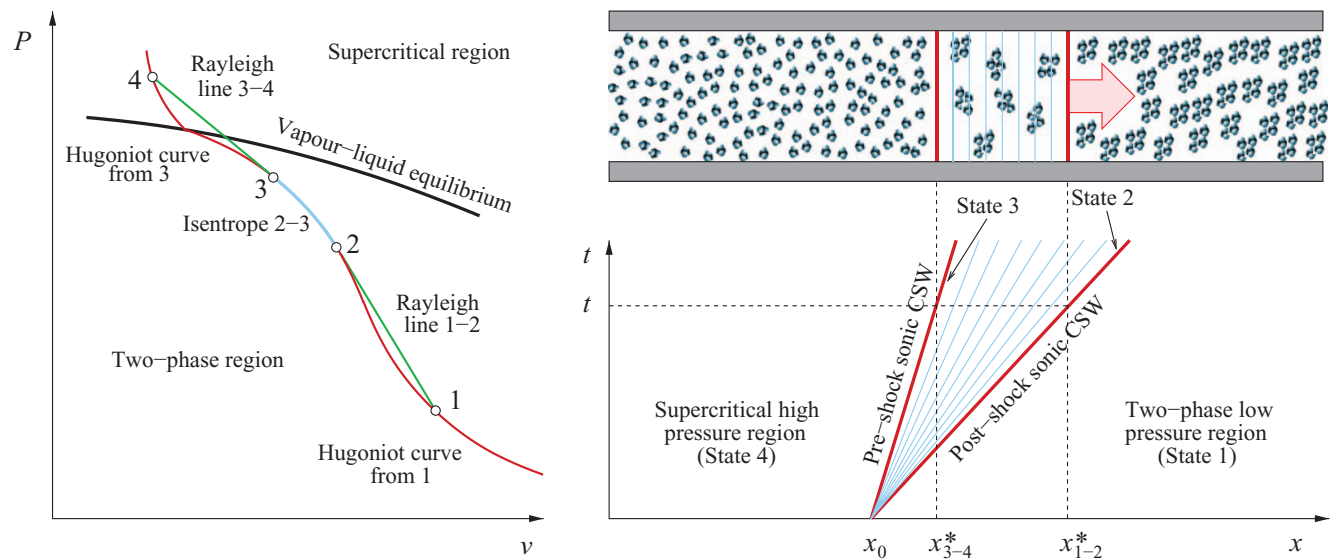

FIG. 4. Example of a composite compression wave displaying a non-classical compression shock wave $(1 \rightarrow 2)$ with a sonic post-shock Mach number $\left(\mathrm{Ma}_{2}=1\right)$, an isentropic compression fan $(2 \rightarrow 3)$, and a second non-classical compression shock wave $(3 \rightarrow 4)$ with a sonic pre-shock Mach number $\left(\mathrm{Ma}_{3}=1\right)$. State 1 is in the vapor-liquid equilibrium region with $\Gamma>0$ and state 4 is in the supercritical or dense-gas region with $\Gamma>0$. Also shown is the $x$ - $t$-plot whereby the compression fan is bounded by two compression shock waves, and a schematic representation (snapshot) of the flow field in a shock tube at time $t=t^{*}$ at the corresponding location. CSW is the abbreviation of compression shock wave. 
It is relevant to the present work that Mujica et al. ${ }^{9}$ reported measurements of the nonlinear acoustic parameter, which is proportional to $\Gamma$, as close as $\pm 0.2 \mathrm{~K}$ to the critical point of $\mathrm{CO}_{2}$, that is, at the reduced temperatures $T / T_{\mathrm{c}}=0.9993$ and 1.007 , and along the three isochores $\rho / \rho_{\mathrm{c}}=0.97,0.95$, and 0.905 . By comparison, the reduced density and temperature of state 1 ahead of the rarefaction shock wave considered in our work are 0.8049 and 0.998 , respectively ( $0.4 \mathrm{~K}$ from the critical point), well within the experimental capabilities demonstrated by Mujica et al. Moreover, in the work by Carlès ${ }^{23}$ predictions from the Navier-Stokes equations complemented with a linearized equation of state are shown to correlate quite well to experimental measurements of acoustic waves generated by thermal inhomogeneities. This physical model is based on the assumption of thermodynamic equilibrium and local homogeneity.

As a final caveat we wish to indicate that, due to phase transition, the shock wave shall be dispersed. In particular, transport properties also exhibit anomalous behavior due to criticality, and the shock-wave thickness is expected to be of the order of a few centimeters, see Ref. 10.

We conclude that according to the predictive scaled fundamental equation of Balfour et al., ${ }^{15}$ two-phase rarefaction shock waves are physically admissible in the close proximity of the vaporliquid critical point. The scaled fundamental equation is based upon the renormalization-group theory applied to the universality class of 3-dimensional Ising-like systems, and therefore it is valid for any system governed by short-range forces. As a consequence, this new fluid-dynamics phenomenon is theoretically expected to occur in all typical fluids. The obtained results are qualitatively independent of the details of the system, e.g., molecular complexity.

This research was supported in part by the Dutch Technology Foundation STW, and the Technology Program of the Ministry of Economic Affairs (Grant No. DSF 11143) and by the Grant No. ADPQ-EN 14 of the Lombardy regional government, Italy.

${ }^{1}$ A. G. Ivanov and S. A. Novikov, "Rarefaction shock waves in iron and steel," Zh. Eksp. Teor. Fiz. 40, 1880 (1961).

${ }^{2}$ P. A. Thompson, G. A. Carofano, and Y. G. Kim, "Shock waves and phase changes in a large-heat-capacity fluid emerging from a tube," J. Fluid Mech. 166, 57 (1986).

${ }^{3}$ W. D. Hayes, in Fundamentals of gasdynamics, High Speed Aerodynamics and Jet Propulsion Vol. 3, edited by H. W. Emmons (Princeton University Press, Princeton, NJ, 1958), pp. 416-481.

${ }^{4}$ R. Menikoff and B. J. Plohr, “The Riemann problem for fluid flow of real materials,” Rev. Mod. Phys. 61, 75 (1989).

${ }^{5}$ A. Chuvatin, A. Ivanov, and L. Rudakov, "Stationary Rarefaction Wave in Magnetized Hall Plasmas," Phys. Rev. Lett. 92, 095007 (2004).

${ }^{6}$ L. Beck, G. Ernst, and J. Gürtner, "Isochoric heat capacity $c_{v}$ of carbon dioxide and sulfur hexafluoride in the critical region,” J. Chem. Thermodyn. 34, 277 (2002).

${ }^{7}$ A. Jounet, B. Zappoli, and A. Mojtabi, "Rapid Thermal Relaxation in Near-Critical Fluids and Critical Speeding Up: Discrepancies Caused by Boundary Effects,” Phys. Rev. Lett. 84, 3224 (2000).

${ }^{8}$ Y. Chiwata and A. Onuki, "Thermal Plumes and Convection in Highly Compressible Fluids," Phys. Rev. Lett. 87, 144301 (2001).

${ }^{9}$ N. Mujica, R. Wunenburger, and S. Fauve, "Scattering of sound by sound in the vicinity of the liquid-vapor critical point," Phys. Rev. Lett. 90, 234301 (2003).

${ }^{10}$ A. Borisov, A. A. Borisov, S. S. Kutateladze, and V. E. Nakoryakov, "Rarefaction shock wave near the critical liquid-vapour point," J. Fluid Mech. 126, 59 (1983).

${ }^{11}$ A. Kluwick, "Adiabatic Waves in the Neighbourhood of the Critical Point," in IUTAM Symposium on Waves in Liquid/Gas and Liquid/Vapour Two-Phase Systems, edited by S. Morioka and L. van Wijngaarden (Kluwer Academic Publishers, 1995), Vol. 31, pp. 387-404.

${ }^{12}$ G. Emanuel, "The fundamental derivative of gas dynamics in the vicinity of the critical point," Technical Report, AME Report 96-1, University of Oklahoma, 1996.

${ }^{13}$ D. S. Kurumov, G. A. Olchowy, and J. V. Sengers, "Thermodynamic properties of methane in the critical region," Int. J. Thermophys. 9, 73 (1988).

${ }^{14}$ J. M. H. Levelt-Sengers, G. Morrison, and R. F. Chang, "Critical behavior in fluids and fluid mixtures," Fluid Phase Equilib. 14, 19 (1983).

${ }^{15}$ F. W. Balfour, J. V. Sengers, M. R. Moldover, and J. M. H. Levelt-Sengers, "Universality, revisions of and corrections to scaling in fluids," Phys. Lett. A 65, 223 (1978).

${ }^{16}$ N. R. Nannan, A. Guardone, and P. Colonna, "On the fundamental derivative of gas dynamics in the vapor-liquid critical region of single-component typical fluids," Fluid Phase Equilib. 337, 259 (2013).

${ }^{17}$ O. Oleinik, "Uniqueness and stability of the generalized solution of the Cauchy problem for a quasi-linear equation," Uspehi Mat. Nauk. 14, 165 (1959).

${ }^{18}$ P. D. Lax, Hyperbolic Systems of Conservation Laws and the Mathematical Theory of Shock Waves (Society for Industrial and Applied Mathematics, Philadelphia, PA, 1973).

${ }^{19}$ C. Zamfirescu, A. Guardone, and P. Colonna, "Admissibility region for rarefaction shock waves in dense gases," J. Fluid Mech. 599, 363 (2008) 
${ }^{20}$ A. Kluwick, "Theory of shock waves. Rarefaction shocks," in Handbook of Shockwaves, edited by G. Ben-Dor, O. Igra, T. Elperin, and A. Lifshitz (Academic Press, San Diego, CA, 2001), Vol. 1, Chap. 3.4, pp. 339-411.

${ }^{21}$ Y. B. Zel'dovich and Y. P. Raizer, Physics of Shock Waves and High-Temperature Hydrodynamic Phenomena (Academic Press, New York, NY, 1966).

${ }^{22}$ A. Guardone, C. Zamfirescu, and P. Colonna, "Maximum intensity of rarefaction shock waves for dense gases," J. Fluid Mech. 642, 127 (2010).

${ }^{23}$ P. Carlès, "Thermoacoustic waves near the liquid-vapor critical point," Phys. Fluids 18, 126102 (2006).

${ }^{24} \mathrm{H}$. Chimowitz, Introduction to Critical Phenomena in Fluids, Topics in Chemical Engineering (Oxford University Press, New York, 2005), p. 368. 